\title{
LII. On the magnetic effects of vibration in iron rods
}

\section{A.O. Rankine D.Sc. \& F.B. Young D.Sc.}

To cite this article: A.O. Rankine D.Sc. \& F.B. Young D.Sc. (1919) LII. On the magnetic effects of vibration in iron rods, Philosophical Magazine Series 6, 38:227, 528-532, DOI: 10.1080/14786441108635981

To link to this article: http://dx.doi.org/10.1080/14786441108635981

曲 Published online: 08 Apr 2009.

Submit your article to this journal $[\pi$

Џ Article views: 3

Q View related articles $₫$ 
where the expansion of the typical S.F. element is

$$
\begin{aligned}
& \sqrt{ }\left(a^{2}-2 a r \mu+r^{2}\right)=\sum\left(\begin{array}{ccc}
r^{n+1} \\
a^{n}
\end{array}, \text { or } \quad \begin{array}{c}
a^{n+1} \\
r^{\prime \prime}
\end{array}\right) I_{n}(\mu), . \\
& \mathrm{I}_{n}(\mu)=\int \mathrm{P}_{n} d \mu=-\underset{n(n+1)}{1-\mu^{2}} d d_{n} d \mu=-\frac{1-\mu^{2}}{n(n+1)} d \frac{d^{2} \mathrm{I}_{n}}{d \mu^{2}} \\
& =\frac{\mathrm{P}_{n+1}-\mu \mathrm{P}_{n}}{n}=\frac{\mu \mathrm{P}_{n}-\mathrm{P}_{n-1}}{n+1}=\frac{\mathrm{P}_{n+1}-\mathrm{P}_{n-1}}{2 n+1} .
\end{aligned}
$$

Dr. Bromwich's recurring relations for his $\mathbf{S}_{n}, \mathbf{S}_{n}{ }^{\prime}$ (Phil. Mag. July, p. 159), are then the equivalent of the definition of $\mathrm{C}_{n}(x)$ in $\$ 1$, or of the differential equation $(5)$ in $\$ 1$, written in a sequence equation, as in (3) $\$ 13$.

I Staple lun, IV.C. I. August 11, 1919.

LII. On the Magnetic Effects of Vibration in Iron Rods. By A. O. Rankine, D.Sce, and F. B. Young, II.Sc."

TN 'Magnetic Induction in Iron and other Metals' (p. 227) 1 Ewing describes an experiment by means of which may be demonstrated the variation in the magnetic state of iron which accompanies mechanical strain. A knowledge of the electrical and magnetic changes produced by mechanical vibration in iron being required in connexion with certain investigations, the following experiments, suggested by Ewing's work, were carried out.

\section{Method of Experiment.}

A cylindrical solid rod $\mathrm{K}$ of mild steel $6 \mathrm{ft}$. long and $1 \mathrm{inch}$ in diameter was clamped at its centre. A conductor of low resistance connected the two ends to form a closed circuit which could, however, be open at will by means of the included switch $S$. At one end of the rod and at right angles to it was fixed a short brass lever L. By striking with a lead bar the lever, the end of the rod or the side of the rod, the latter could be thrown into torsional, longitudinal or hateral vibration respectively.

Observations were made with a small search-coil G, 5 inches

* Communicrted by Professor J. C. McLemnan, Ph.D., F.R.S. 
Magnetic Effects of Vibration in Iron Rods.

square, consisting of about 200 turns of insulated copper wire and connected through a three-valve amplifier $\mathrm{A}$ to

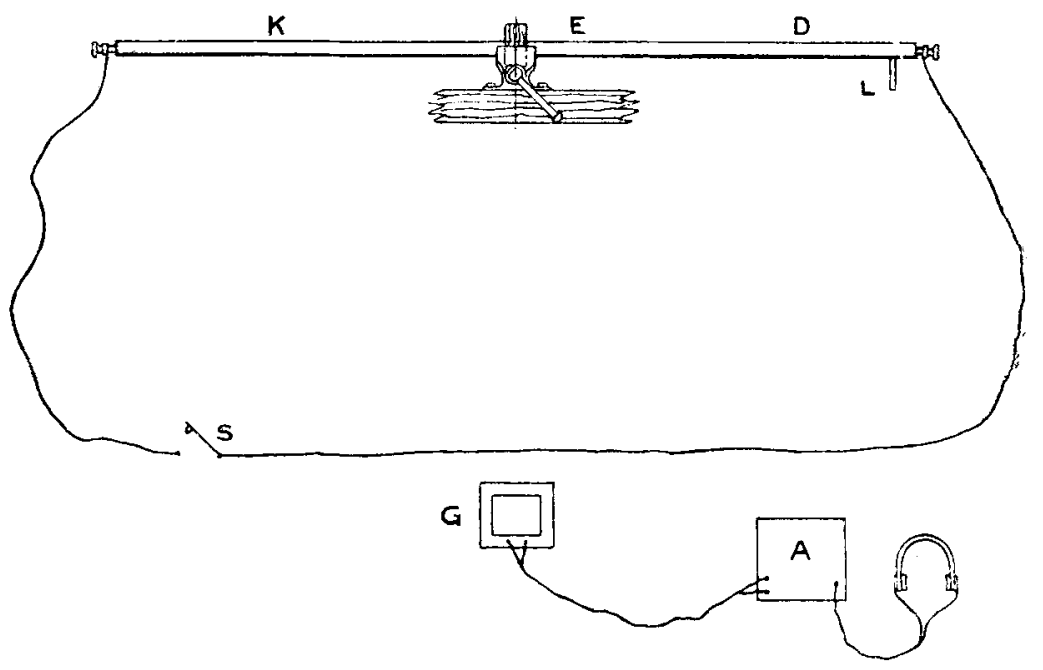

telephone receivers. The coil was brought near the cable or rod in three typical positions:-

Position C. The coil in the same plane as the conductor; in this position it would detect any oscillating field having a component whose lines are concentric with the conductor.

Position P. Axis of coil parallel to conductor ; in this position it would detect any oscillating field having a component whose lines are parallel to the conductor.

Position R. Axis of coil at right angles to and passing throngh the conductor; in this position it would detect any component whose lines are radial to the conductor.

During the observations the rod was placed roughly in the magnetic meridian. The orientation of the bar, bowever, did not appear to have much influence on the character of the observation.

\section{General Observations.}

The following observations apply equally to all modes of vibration of the bar.

(a) Coil near cable.

When the coil was placed near the cable in position $C$, 
sounds were clearly heard in the telephones corresponding to the musical "clink" of the rod when struck. These sounds, however, died out as the coil was rotated to either position $\mathrm{P}$ or position $\mathrm{R}$. 'The disturbances were thus clearly due to an oscillating electric current induced in the cable by magnetic changes produced in the rod; such a current would produce a magnetic field around the cable whose lines would be concentric with the cable and would have no parallel or radial component.

If the circuit was broken the sounds in the telephone disappeared entirely, as would be expected.

(b) Coil near Roul.

When the coil was held near the rod the vibrations were audible in the telephones as before. The sounds were not, however, cut out by the rotation of the coil; they were in fact sometimes greater in intensity for position $\mathrm{P}$ or $\mathrm{R}$ than for position C. Definite differences of quality were perceptible as the coil rotated, though the fundamental tone remained unaltered.

If the circuit was broken the sounds in the telephonereceivers persisted, though with certain changes of intensity and quality.

(c) Observations with two Search-Coils.

Two similar search-coils were connected in series with the amplifier.

(i.) These were placed near different parts of the cable in position C. By rotating one of these it was possible to balance the e.m.t.'s induced in the two coils so that silence was produced.

(ii.) Attempts were made to balance one coil near the cable against the other placed near the rod. Only a slight reduction in the intensity of the sound could, however, be produced, indicating that the e.m.f.'s induced in the two coils were not in phase.

(iii.) The two coils were next placed near different parts of the rod, both in position C. Since rotation of either coil would have introduced some component of the magnetic field other than the concentric, an attempt was made to balance the induced e.m.f.'s by turning the coils into opposition and then moving each in turn, without rotation, further from the rod. No signs of even partial neutralization were, however, perceived. Hence the magnetic oscillation at two different parts of the rod were ont of phase. 
The oscillating magnetic field around the rod was obviously far less simple in character than that round the cable.

\section{Observations of various modes of Vibration.}

The following observations relate to particular modes of vibration produced as described above. In each case the frequency of the note transmitted directly through the air was determined by means of a sonometer and standard tuning-fork. This was compared with the frequency given by calculation, a rough verification of the mode of vibration being thus obtained.

(a) Torsional Vibration.

The note heard from the bar was $950 \sim /$ sec. (calculated frequency about $880 \sim / \mathrm{sec}$.), and the same note was detected in the receivers.

Near the rod on closed circuit the concentric, parallel and radial components could all be detected, their relative intensities varymg at different points along the bar. Breaking the eircuit produced no marked change in the field around the rod except in the case of the concentric component. In this case a marked diminution was observed near the end of the rod, but very little near the clamped centre. The observation suggests that the concentric component near the end was largely sucondary and due to the current produced in the circuit; whilst that near the centre was directly associated witb the magnetic state of the rod.

(b) Longitudinal Vibrations.

The note heard directly from the rod had a frequency of $1400 \sim /$ sec. (calculated frequency about $1400 \sim /$ sec.), and the same note was detected in the receivers.

The variation in the strengths of the radial and parallel components at various parts of the rod suggested the exjstence of a field such as is commonly associated with a rod longitudinally magnetized. 'Thus at $\mathrm{D}$ the radial component was stronger than the parallel component, whilst at $\mathrm{E}$ the parallel component was the stronger though it was weaker than the radial component at $D$. The effects observed when the coil was threaded on the bar were consistent with this view; the longitudinal note was clearly heard and was loudest when the coil was near the centre of the bar.

(c) Lateral Vibration.

By striking the rod at suitable points il was possible to emphasize various overtones which were identified by means 
of the sonometer with the calculated values. The following table shows $(a)$ the calculated frequencies of the fundamental and first overtones ; $(b)$ the frequencies detected by listening directly to the bar; (c) the frequencies detected in the telephones when the coil was placed near the rod.

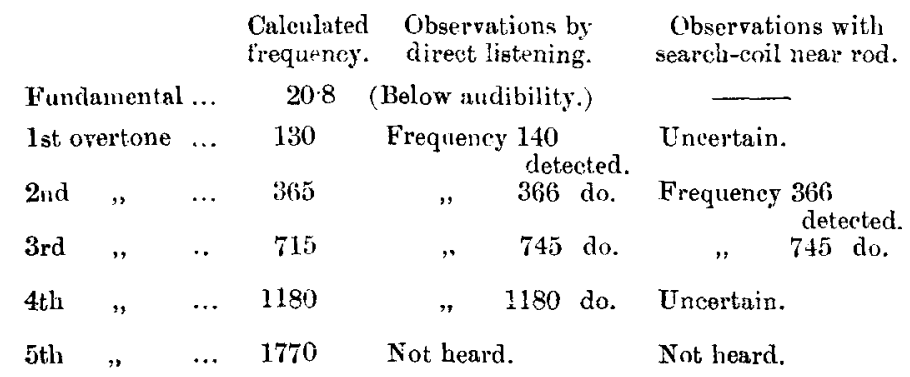

To obviate the damping effects due to the cable, this was disconnected from the bar during the foregoing observations.

It will be observed that though the first and fourth overtones were distinguished by direct listening; they could not be distinguished with certainty in the receivers. They may have been present, but were masked by the more prominent second and third overtones which could not be suppressed.

As the coil was rotated between the positions $C, P$, and $R$ variations of quality were observed, and the concentric component was in general less intense than the parallel or radial components.

\section{Conclusions.}

'The phenomena observed indicate that complex magnetic changes are produced in an iron bar by its own vibrations.

Though the mode of vibration of the har determines the period of its vibration and therefore the period of the magnetic oscillations w hich result therefrom, no very simple and detinite relation exists between the mode of vibration and the structure of the resulting magnetic field. (Longitudinal vibration seemed, however, to be associated with a marked oscillation in the longitudinal maguetization.)

It may be noticed as a point of practical importance that such " magneto-mechanical" effects may lead to disturbances in sonsilive electrical circuits which pass near moving steel machinery unless those circuits are shielded or rendered noninductive. 